\section{A INFLUÊNCIA DA SOBRECARGA DE TRABALHO DO ENFERMEIRO NA QUALIDADE DA ASSISTÊNCIA PRÉ-NATAL}

\author{
The influence of nurse's work overload on the quality \\ of prenatal care
}

\section{La influencia de sobrecarga de trabajo del enfermero en la calidad de la atención prenatal}

\section{RESUMO}

Objetivo: Verificar se a sobrecarga de trabalho do enfermeiro influencia na consulta prénatal prestada às gestantes, sob a visão de ambos. Métodos: Estudo descritivo e qualitativo, realizado em Guarapuava/PR, em 2010, investigou 10 gestantes de baixo risco e 10 enfermeiras responsáveis de 5 unidades básicas de saúde, utilizando entrevista com questionário semiestruturado, contendo caracterização da amostra e questões sobre o atendimento pré-natal recebido e prestado. Os dados receberam análise de conteúdo. Resultados: Os enfermeiros tem entre 25 e 47 anos, possuem de 3 a 21 anos de formados e o tempo de trabalho na unidade variou de 6 meses a 7 anos. As gestantes possuíam entre $21-30$ anos, são solteiras, multíparas e frequentam a unidade há mais de 1 ano. Os enfermeiros relatam possuir sobrecarga de trabalho que influencia negativamente no atendimento pré-natal à gestante e o número de atividades realizadas ultrapassa o tempo que dispõem para executá-las. As gestantes exibiram divergência de opinião, ficando evidente que o atendimento poderia ser melhorado, e que o pouco tempo dispendido para os atendimentos interfere na qualidade. Conclusão: A sobrecarga de trabalho para o enfermeiro influencia na qualidade da assistência que é prestada às gestantes. $\mathrm{E}$ as gestantes percebem que a baixa qualidade do atendimento prestado por este profissional está ligada ao grande número de atividades que esse precisa desenvolver.

Descritores: Enfermagem do Trabalho; Jornada de Trabalho; Cuidado Pré-Natal; Gestantes.

\section{ABSTRACT}

Objective: To check whether nurse's work overload influences prenatal care provided to pregnant women according to both point of views. Methods: Descriptive and qualitative study conducted in 2010 in Guarapuava/PR with 10 low-risk pregnant women and 10 nurses in charge of 5 basic health care units. Interviews were carried out using a semi-structured questionnaire containing the characterization of the sample and questions on prenatal care received and provided. Data underwent content analysis. Results: Nurses are in the age group between 25 and 47 years, have 3-21-year experience after graduation and the time spent at the job ranged from 6 months to 7 years. Pregnant women were between 21-30 years old, single, multipara and have been attending the unit for more than one year. Nurses report work overload that negatively affects the prenatal care provided to pregnant women, and the number of activities carried out exceed the time they have to perform them. Pregnant women presented different opinions and it became evident that the care could be improved and that the short time spent on the consultations interferes in the quality. Conclusion: For the nurse, the work overload influences the quality of care provided to pregnant women, and pregnant women notice that the poor quality of care provided by this professional is associated with the large number of activities they need to perform.

Descriptors: Occupational Health Nursing; Work Hours; Prenatal Care; Pregnant Women.
Artigo Original

\section{Danielle Wisniewski ${ }^{(1)}$ Gleisy Gróss ${ }^{(2)}$ Rosely Bittencourt ${ }^{(3)}$}

1) Universidade Estadual do Centro-Oeste UNICENTRO - Guarapuava (PR) - Brasil

2) Enfermeira, graduada pela Universidade Tuiuti do Paraná - UTP - Curitiba (PR) -

Brasil

3) Instituto Tecnológico e Educacional ITECNE - Curitiba (PR) - Brasil
Recebido em: 27/06/2013

Revisado em: 26/08/2013 Aceito em: 18/11/2013 


\section{RESUMEN}

Objetivo: Verificar si la sobrecarga de trabajo del enfermero influye en la consulta de prenatal ofrecida a las mujeres embarazadas a partir de ambos puntos de vista. Métodos: Estudio descriptivo y cualitativo realizado en Guarapuava/PR en 2010 con 10 mujeres embarazadas de bajo riesgo y 10 enfermeras responsables por 5 unidades básicas de salud a través de una entrevista con cuestionario semi-estructurado con la caracterización de la muestra y cuestiones de la atención prenatal ofrecida y recibida. Los datos fueron analizados a través del análisis de contenido. Resultados: Los enfermeros tienen entre 25 y 47 años, entre 3 y 21 años de licenciatura y el tiempo de trabajo en la unidad varió entre 6 meses y 7 años. Las mujeres embarazadas tenian entre 21 y 30 años, solteras, multíparas y frecuentan la unidad hace más de un año. Los enfermeros relatan una sobrecarga de trabajo que influye de manera negativa en la atención prenatal de las mujeres embarazadas y el número de actividades realizadas sobrepasa el tiempo que tienen para hacerlas. Las mujeres embarazadas mostraron opiniones distintas quedándose evidente que la atención prenatal podría ser mejor y que el poco tiempo utilizado para ella influye en su calidad. Conclusión: La sobrecarga de trabajo del enfermero influye en la calidad de la atención ofrecida a las mujeres embarazadas. Las mismas perciben que la baja calidad de la atención de parte de este profesional está asociada al gran número de actividades que el mismo necesita realizar.

Descriptores: Enfermería del trabajo; Horas de trabajo; Atención prenatal; Mujeres embarazadas.

\section{INTRODUÇÃO}

O pré-natal tem como principal objetivo acolher a mulher desde o início da gestação, garantindo uma gravidez segura e o nascimento de uma criança saudável. Para uma assistência qualificada e humanizada, é preciso adotar condutas acolhedoras e sem intervenções desnecessárias, de modo a permitir acesso aos serviços de saúde de qualidade, com ações integradas em todos os níveis da atenção, desde promoção, prevenção e assistência à saúde da gestante e do recém-nascido, até o atendimento ambulatorial básico e atendimento hospitalar de alto risco ${ }^{(1)}$.

De acordo com o Programa de Humanização no PréNatal e Nascimento, regulamentado pela Portaria/GM n ${ }^{\circ}$ 569 , de 1/6/2000, uma de suas prioridades é adotar ações que assegurem a melhoria do acesso, da cobertura e da qualidade do acompanhamento pré-natal, da assistência ao parto, assistência neonatal e puerperal ${ }^{(2)}$.

No contexto da porta de entrada do cliente no sistema de saúde, a Atenção Básica tem a Estratégia Saúde da Família (ESF) como prioridade para sua organização, e conforme preceitos do Sistema Único de Saúde (SUS), caracterizase como um conjunto de ações de saúde, individuais e coletivas, que abrangem a promoção e a proteção da saúde, a prevenção de agravos, o diagnóstico precoce, o tratamento, a reabilitação e a manutenção da saúde ${ }^{(3)}$.

$O$ trabalho da enfermeira na ESF integra o acompanhamento das condições de saúde, no atendimento individual ou no atendimento em grupo; no levantamento e monitoramento de problemas de saúde, sendo um enfoque de risco ou de vulnerabilidade, articulando ações à intervenção nos agravos de ordem patológica, e no exercício de uma prática de enfermagem comunicativa, quanto a riscos e cuidados, auxiliando no conhecimento e autonomia dos sujeitos. Sendo assim, nota-se que as práticas de enfermagem pressupõem o entendimento de que a enfermagem, enquanto prática social, busca responder às necessidades sociais e de saúde de determinado tempo e espaço social( ${ }^{(4)}$.

Pode-se perceber que as enfermeiras possuem inúmeras funções enquanto profissionais integrantes da equipe de ESF, mas aponta-se que há precarização do trabalho de enfermagem, o qual acarreta sobrecarga física e psíquica, pois atuam com pouca possibilidade de desenvolver o trabalho seguindo os parâmetros profissionais, dificultando a utilização da Sistematização da Assistência de Enfermagem (SAE), que trata da organização das informações referentes às atividades desenvolvidas pelo profissional enfermeiro em favor de uma assistência individual, integral e resolutiva ao paciente, e algumas vezes com desvio de função ${ }^{(5)}$.

Vale ressaltar que o objetivo do acompanhamento prénatal é assegurar o desenvolvimento da gestação, permitindo o parto de um recém-nascido saudável, sem impacto para a saúde materna, inclusive abordando aspectos psicossociais. A partir da avaliação da necessidade de cada usuária, o acesso a outras redes assistenciais deve ser garantido às gestantes pelo enfermeiro através do rastreamento dos sintomas e situações de risco, como a oferta de atendimento clínico e psicológico à vítima de violência, ou àquela em risco de depressão pós-parto, fazendo com que o profissional enfermeiro precise estar disponível desde a gestação até o puerpério( ${ }^{(6)}$. No Brasil, a adesão ao Pré-Natal tem se mostrado presente em $75 \%$ das gestantes, o que é fator favorecedor para um parto sem intercorrência e o nascimento de um recém-nascido saudável ${ }^{(6)}$.

Dado que a demanda dos serviços públicos de saúde aumenta a cada dia, existe influência no acúmulo de atividades, e, consequentemente, sobrecarga de trabalho do profissional enfermeiro. Sendo o pré-natal mais uma atividade desempenhada por este profissional, no presente estudo questionou-se se a sobrecarga de trabalho do profissional enfermeiro influencia na assistência pré-natal que é prestada à gestante.

Isto posto, o presente estudo objetivou verificar se a sobrecarga de trabalho do enfermeiro influencia na consulta pré-natal prestada às gestantes, sob a visão de ambos. 


\section{MÉTODOS}

Esta pesquisa norteou-se como um estudo de campo, do tipo descritivo de abordagem qualitativa. A metodologia foi escolhida, pois pesquisas qualitativas são capazes de incorporar a questão do significado e da intencionalidade como inerentes aos atos, às relações sociais, e às estruturas sociais $^{(7)}$.

Realizou-se o presente estudo no município de Guarapuava/PR, localizado no centro-sul do estado do Paraná, cuja população correspondia a 167.328 habitantes no ano de 2010, distribuídos em uma área territorial de $3.117,011 \mathrm{~km}^{2}$. Neste município há 95 estabelecimentos de saúde, distribuídos em 51 privados, 42 municipais e 2 estaduais, inexistindo federal ${ }^{(8)}$.

Investigaram-se 10 gestantes de baixo risco de 5 unidades básicas de saúde (UBS) (Clínica da Mulher, Centro Integrado de Atendimento Santa Cruz, Vila Carli, Xarquinho e Santana), sendo 2 gestantes de cada unidade e as enfermeiras responsáveis. Essas UBS selecionadas atendem cerca de $70 \%$ das gestantes do município. Já quanto às gestantes investigadas, sua seleção ocorreu por meio de um sorteio do total das presentes nas UBS no dia da coleta de dados; enquanto as enfermeiras eram as únicas profissionais da enfermagem de cada UBS.

Seguiram-se os seguintes critérios de inclusão: ser gestante de baixo risco correspondente à faixa etária 2040 anos, ter realizado duas ou mais consultas pré-natal anteriores à coleta de dados. Quanto à enfermeira, esta deveria ser a responsável pela UBS e ter realizado a consulta com a paciente.

A coleta de dados foi realizada durante o mês de novembro de 2010 por pesquisadora treinada, obtendo-se as informações diretamente com as duas gestantes de cada UBS e a enfermeira responsável, através de entrevista, com duração de cerca de 30 minutos, realizada após a consulta pré-natal com o profissional enfermeiro, sendo gravado o relato das entrevistadas. As gestantes e as enfermeiras responderam a um questionário semi-estruturado, composto de perguntas abertas e fechadas, direcionado às gestantes, com questões para caracterização da amostra e sobre o atendimento pré-natal recebido; e, ainda, sobre o atendimento pré-natal prestado, quando questionado às enfermeiras.

Realizou-se a análise do material de acordo com Análise de Conteúdo ${ }^{(9)}$, e para preservar a identificação das participantes, utilizaram-se codinomes de flores para as enfermeiras e codinomes de cores para as gestantes.

Os dados obtidos passaram pela pré-análise, seguida de leitura flutuante e atribuição de um código a cada um dos sujeitos; e posteriormente, leitura detalhada do texto transcrito, codificação do material, seguido de categorização, interpretação do conteúdo e discussão com os dados encontrados ${ }^{(9)}$, sendo que desta análise emergiram as seguintes categorias: 1. Sobrecarga de Atividades dos Profissionais Enfermeiros; e 2. Qualidade no Atendimento Pré-Natal.

O estudo recebeu aprovação pelo Comitê de Ética em Pesquisa (CENECT - Centro Integrado de Educação Ciência e Tecnologia S/C - PR), sob o protocolo $n^{\circ}$ 098/10.

\section{RESULTADOS E DISCUSSÃO}

Neste espaço serão apresentados os dados de identificação dos sujeitos da pesquisa e, em seguida, apresentar-se-ão as categorias temáticas.

\section{Dados de identificação dos sujeitos do estudo}

Dentre os profissionais que participaram do estudo, um é do sexo masculino e o restante (9) do sexo feminino; situam-se na faixa etária compreendida entre 25 e 47 anos, possuem de 1 a 21 anos de formados em enfermagem; o tempo de trabalho na unidade variou de no mínimo há 6 meses e no máximo há 7 anos. Com relação à qualificação profissional, três enfermeiros não possuíam nenhum tipo de especialização, uma possuía especialização na área de Saúde Pública e outra em Auditoria. Recrutados do próprio município, são trabalhadores com carga horária semanal de 40 horas, cujo salário variou entre $\mathrm{R} \$ 1.300,00$ a $\mathrm{R} \$$ $2.500,00$, apresentavam mais de um vínculo empregatício com realização de plantões no SAMU (Serviço de Atendimento Móvel de Urgência) da cidade com ganho de horas-extra, bem como, também trabalhavam como docentes em curso técnico profissionalizante.

As gestantes que colaboraram com a pesquisa possuem entre 21 - 30 anos (07) e seis são solteiras; sete são multigestas e frequentam a unidade há mais de um ano.

A seguir apresentam-se as categorias que emergiram deste estudo.

\section{Sobrecarga de Atividades dos profissionais enfermeiros}

Os enfermeiros possuem inúmeros atributos enquanto integrantes da equipe de saúde, podendo-se salientar que a elevada carga de atividades que precisam ser realizadas, muitas vezes, ultrapassa o tempo do qual eles dispõem para executá-las. Esta informação é referendada pelas seguintes afirmações:

"Muitas vezes tenho tanta coisa pra fazer, que acabo não fazendo nada direito [...]" (Lírio)

"Alguns pacientes vão embora, pois não têm tempo para esperar e falar comigo [...] é uma pena" (Rosa) 
"Quando está nos dias de entregar relatório, não tenho tempo nem para ir ao banheiro, é muita coisa para fazer [...]" (Violeta)

"Não realizo grupo de gestantes, não dá tempo o suficiente para ficar parada lá só conversando com elas, os agentes comunitários orientam, quando elas possuem dúvidas [...]" (Tulipa)

Face ao exposto pelas falas das enfermeiras, é notável que o tempo que as enfermeiras dispõem para realizar as diversas atividades é escasso. Em um estudo ${ }^{(10)}$ com 5 enfermeiras atuantes no ambulatório de um Hospital Universitário, ao avaliar sobre a consulta de enfermagem e as ações educativas no pré-natal, concluíram que a ação educativa realizada pela enfermeira durante a consulta do pré-natal caracteriza-se como uma ação de rotina, sendo pouco participativa, com predominância informativa. Este mesmo estudo mostra que a consulta, apesar da existência da ideia em educar, apresenta questões relacionadas ao modelo assistencial, estrutural e organizacional da instituição, que se tornam obstáculos para a realização da educação em saúde, sugerindo uma reorientação do serviço de enfermagem na atenção à gestante, o surgimento de um ambiente físico adequado para o atendimento da consulta de enfermagem e a participação da gestante em grupos ${ }^{(10)}$.

A falta de tempo das profissionais, encontrada no presente estudo, corrobora com um estudo que objetivou identificar e analisar os atributos mobilizados pela enfermeira no cotidiano na Saúde da Família voltados ao planejamento, coordenação e supervisão, visando uma aproximação aos desempenhos, e revelou que as enfermeiras desenvolvem o planejamento de ações para a organização do cuidado juntamente com o exercício da coordenação da Unidade Básica, o que resulta em sobrecarga de trabalho ${ }^{(11)}$.

Pelas colocações dos enfermeiros, e referendada pela fala de Margarida, a presente pesquisa pode corroborar com a afirmação de que a ação do pré-natal não possui ação educativa, sendo mecânica e rotineira ${ }^{(10)}$.

"Atendo as gestantes só na quinta-feira à tarde, cada consulta demora uns 10 minutos, às vezes demora um pouco mais, quando elas tem dúvidas ou quando saio para ver outras coisas, e dai demoro um pouco para voltar[...]" (Margarida)

Foi possível constatar que as gestantes também notam a sobrecarga de trabalho do profissional enfermeiro, conforme se verifica nas falas a seguir:

"Sempre que eu preciso falar com a enfermeira, tenho que esperar pelo menos uns 30 minutos. Ela nunca me atende quando eu chego. Sempre está correndo, pra lá e pra cá $[\ldots]$ ". (Azul)
"Várias vezes em que a enfermeira está me examinando, ou a gente está conversando, ela precisou sair, pois a estavam chamando para ver outras coisas [...]”. (Amarelo)

"Teve um dia que não tinha médico no postinho, e eu tive que ir na urgência, porque tinha muita gente antes de mim para falar com a enfermeira, e eu estava com muita dor nas costas [...]”. (Lilás)

Os pacientes conseguem notar essa falta de tempo dos profissionais, o que ocasiona reclamações por parte dos usuários do serviço. Diferentemente do que é focado como prioridade da Política que engloba essa população. Em uma pesquisa que avaliou o Programa de Humanização do PréNatal e Nascimento, mostrou-se que há diferenças entre as recomendações e os desejos e necessidades da mulher com os objetivos do Programa, fazendo com que a gestante trace para si outro fluxo de atendimento. Esta ocorrência acaba trazendo prejuízos ao vínculo que estabelece com o serviço de saúde, além de dificuldades de controle pelo serviço do seguimento real que está sendo oferecido. Numa reflexão realizada pelos autores do programa, tomando por base a perspectiva das mulheres atendidas, identificou aspectos cuja consideração no momento da avaliação poderia resultar em maior efetividade e humanização do controle pré-natal oferecido $^{(12)}$.

\section{Qualidade no atendimento Pré-Natal}

No que tange ao termo qualidade, o mesmo é definido ${ }^{(13)}$ como o alcance dos resultados esperados pela empresa e satisfação dos consumidores dos serviços prestados. Já no que tange à qualidade em saúde, o seu alcance é obtido ao se relacionar os maiores benefícios possíveis, associados aos menores riscos para o paciente, por meio de recursos desejáveis $^{(14)}$.

A Estratégia Saúde da Família busca a excelência em qualidade no atendimento dos pacientes, orientandose pelos mesmos princípios do SUS, se concretizando através de ações gerenciais, sanitárias, democráticas e participativas, com o trabalho em equipe, referentes a populações de territórios bem definidos; usa tecnologias de elevada complexidade e baixa densidade, para resolver os problemas de saúde mais comuns em seu território, sendo o contato preferencial dos usuários com os sistemas de saúde $^{(3)}$.

No momento em que os profissionais são questionados sobre a definição de qualidade no atendimento prestado, eles demonstram-se imprecisos na resposta.

\footnotetext{
"É dar ao paciente o que ele precisa?[...]” (Rosa)

"Se você resolver os problemas deles, já está bom [...]" (Margarida)

"Tem que fazer as coisas bem feitas [...]” (Lírio)
} 
Está evidente nas falas anteriores o descaso aparente com a assistência que é prestada às pacientes, faz com que essas apresentem maior evasão das consultas pré-natais, podendo gerar complicações para a mãe e também para o feto. Em um estudo ${ }^{(15)}$ da Qualidade da assistência ao trabalho de parto pelo Sistema Único de Saúde, as gestantes de baixo risco são submetidas a intervenções desnecessárias e as de alto risco não recebem cuidado adequado. Como consequência, os resultados perinatais são desfavoráveis e as taxas de cesariana e de mortalidade materna são incompatíveis com os investimentos e a tecnologia disponível.

Em outro estudo ${ }^{(16)}$, avaliou-se a Qualidade assistencial na ótica de enfermeiros, na Divisão de Enfermagem MaternoInfantil de um Hospital Universitário, o qual evidenciou que na perspectiva da melhoria contínua da qualidade, é de extrema importância identificar os problemas, no intuito de implantar ações eficazes, monitorar seu processo e execução, e, finalmente, favorecer o trabalho de avaliação dos serviços, para conseguir o padrão ideal de excelência da qualidade. A gestão da qualidade demanda diversas estratégias e ferramentas, mas é preciso a colaboração e comprometimento das pessoas, pois, os colaboradores são as pessoas diretamente envolvidas no fluxo e dinâmica de trabalho, traduzindo o potencial do grupo ${ }^{(16)}$.

“Acho que é boa, quando dá tempo oriento e esclareço dúvidas, mas se o tempo é curto ou tem muita gente para atender, eu escuto o bebê e oriento os cuidados mais importantes [...]" (Rosa)

"Eu considero que é muito boa, me esforço para fazer o melhor possivel, mas nem sempre é o que acontece [...]" (Tulipa)

"Se eu tivesse mais tempo, seria muito melhor. Hoje faço como dá, depende da demanda e as intercorrências que surgem [...]" (Lírio)

As gestantes exibiram divergência de opinião, quando foram questionadas sobre a qualidade do atendimento que lhes é prestada, ficando evidente que $\mathrm{o}$ atendimento poderia ser melhorado, e que o pouco tempo dispendido para os atendimentos interfere na qualidade, como se percebe nas falas a seguir:

\footnotetext{
"Acho que é bom, mas poderia ser melhor, [...], deviam ter mais tempo pra gente" (Vermelho)

"Acho ruim, tenho muitas dúvidas que não dá tempo para esclarecer, [...]" (Cinza)

"Gosto bastante, a enfermeira é muito querida, mas tem vezes que ela só escuta o meu bebê e vê os meus exames, mas eu gosto. Ela que sabe o que é importante não é?" (Verde)
}

As gestantes queixam-se da qualidade do atendimento, mas de modo subjetivo, o que dificulta a avaliação clara do atendimento recebido, deste modo seria possível planejar a elaboração de um instrumento para avaliar a qualidade do atendimento pré-natal recebido pela gestante, e promover a discussão com gestores, de modo a favorecer a implantação deste, e garantir uma melhor qualidade do atendimento. É importante salientar que é necessário o planejamento de ações educativas para o desenvolvimento do enfermeiro com capacidade crítica e reflexiva perante as atividades que precisa desenvolver, e também que auxilie no processo de efetivação do Programa Nacional de Humanização do Parto e Nascimento, que garantiria uma melhor qualidade no atendimento prestado, e também na redução das taxas de mortalidade materno-infantil ${ }^{(17)}$.

\section{CONSIDERAÇÕES FINAIS}

No presente estudo conseguiu-se mostrar que a sobrecarga de trabalho para o enfermeiro que atua em atenção básica influencia na qualidade da assistência que é prestada às gestantes. E as gestantes avaliadas percebem que a baixa qualidade do atendimento prestado por este profissional está ligada ao grande número de atividades exercidas por ele.

É necessária uma maior dedicação por parte dos profissionais, para atender as gestantes de sua área, e incentivar às consultas de pré-natal. As gestantes respondem às ofertas de atenção conforme o que pensam sobre suas necessidades de saúde. A potencialização dessas informações nas ações avaliativas mostra a inclusão de diferentes sujeitos de um programa como "sujeitos de autoavaliação" e não apenas como "alvo analítico", resultando tanto em uma aproximação mais realista das necessidades de saúde da população como em uma oportunidade de refletir e fortalecer esse ator. Sendo assim, quando uma equipe de saúde não se preocupa com a criação de vínculo com a gestante, aumenta-se o risco de desistência ou de menor frequência no acompanhamento. A gestante se motiva a buscar aquelas que lhe tragam maior segurança, em um movimento natural de obter as melhores condições possíveis para o acompanhamento do crescimento e desenvolvimento de seu filho.

Propõe-se a elaboração de outros estudos relacionados à sobrecarga de trabalho enfrentada pelo profissional enfermeiro, com o intuito de mostrar a necessidade de contratação de um maior número de profissionais, e consequente melhora na qualidade das consultas de enfermagem realizadas. Sendo melhor também para os pacientes atendidos.

O estudo sugere uma reorientação do serviço de enfermagem na atenção à gestante, o surgimento de um 
ambiente físico adequado para o atendimento da consulta de enfermagem e a participação da gestante em grupos de apoio.

\section{REFERÊNCIAS}

1. Ministério da Saúde (BR). Pré-natal e Puerpério: atenção qualificada e humanizada: manual técnico. Brasília: Ministério da Saúde; 2005. (Série Direitos Sexuais e Direitos Reprodutivos, $\mathrm{n}^{\circ}$ 5).

2. Ministério da Saúde (BR). Programa Humanização do Parto: humanização no pré-natal e nascimento. Brasília: Ministério da Saúde; 2002. (Série C. Projetos, Programas e Relatórios, $n^{\circ} 43$ ).

3. Ministério da Saúde (BR). Política nacional de atenção básica. Brasília: Ministério da Saúde; 2006. (Série Pactos pela Saúde 2006, v. 4).

4. Ermel RC, Fracolli LA. O trabalho das enfermeiras no Programa de Saúde da Família em Marília/SP. Rev Esc Enferm USP. 2006;40(4):533-9.

5. Bertoncini JH, Pires DEPP, Scherer MDA. Condições de trabalho e renormalizações nas atividades das enfermeiras na saúde da família. Trab Educ Saúde. 2011;9(Supl 1):157-73.

6. Ministério da Saúde (BR), Secretaria de Atenção à Saúde, Departamento de Atenção Básica. Atenção ao pré-natal de baixo risco. Brasília: Ministério da Saúde; 2012.

7. Minayo MCS. O desafio do conhecimento: pesquisa qualitativa em saúde. $8^{\text {a }}$ ed. São Paulo: HUCITEC; 2004.

8. Instituto Brasileiro de Geografia e Estatística - IBGE. Censo demográfico 2009/2010. Rio de Janeiro; 2010 [acesso em 2010 Fev 22]. Disponível em: http://www. ibge.gov.br /home/presidencia/noticias /imprensa/ppts/ 00000008473104122012315727483985.pdf

9. Bardin L. Análise de conteúdo. Lisboa: Edições 70; 2009.
10. Kawata LS, Mishima SM, Chirelli MQ, Pereira JB. O trabalho cotidiano da enfermeira na saúde da família: utilização de ferramentas da gestão. Texto Contexto Enferm. 2009;18(2):313-20.

11. Almeida CAL, Tanaka OY. Perspectiva das mulheres na avaliação do Programa de Humanização do Pré-Natal e Nascimento. Rev Saúde Pública. 2009;42(1):98-104.

12. Balsanelli AP, Jericó MC. Os reflexos da gestão pela qualidade total em instituições hospitalares brasileiras. Acta Paul Enferm. 2005;18(4):397-402.

13. Donabedian A. Quality assurance in health care: consumers' role. Qual Health Care. 1992;1(4):247-51.

14. Oliveira MIC, Dias MAB, Cunha CB, Leal MC. Qualidade da assistência ao trabalho de parto pelo Sistema Único de Saúde, Rio de Janeiro (RJ), 19992001. Rev Saúde Pública. 2008;42(5):895-902

15. Yuri NE, Tronchin DMR. Qualidade assistencial na divisão de enfermagem materno-infantil de um hospital universitário na ótica de enfermeiros. Rev Esc Enferm USP. 2010;44(2):331-8

16. Patah LEM, Malik AM. Modelos de assistência ao parto e taxa de cesárea em diferentes países. Rev Saúde Pública. 2011;45(1):185-94.

17. Brondani JE, Aranda AL, Morin VL, Ferraz TR, Marques CL, Colomé EF. Percepções de gestantes e puérperas acerca da sala de espera em uma unidade básica de saúde integrada à estratégia saúde da família. Rev Bras Promoç Saúde. 2013;26(1):63-70.

\section{Endereço para correspondência:}

Danielle Wisniewski

Departamento de Enfermagem da Universidade Estadual

do Centro-Oeste (UNICENTRO).

Rua Luis Cavalcanti da Silva, 182

CEP: 85045-380 - Guarapuava - PR - Brasil

E-mail: daniwisni@gmail.com 